Optical properties of suspensions of gold half-shells

\title{
Optical properties of suspensions of gold half-shells
}

Jingquan Liu, Katie E. McBean, Nadine Harris and Michael Cortie

Institute for Nanoscale Technology, University of Technology Sydney, PO Box 123, Broadway, NSW 2007, Australia

Corresponding author : Prof. Michael Cortie

e-mail : michael.cortie@uts.edu.au

phone : +61-2-9514-2208

\begin{abstract}
Suspensions of mesoscale gold half-shells of controlled size were produced by microsphere-templated vapour deposition and their optical properties studied. The transmission spectra of the particles exhibited an extinction peak that could be tuned from $530 \mathrm{~nm}$ to over $2000 \mathrm{~nm}$ by variation of the diameter of the template used. In this respect the optical properties of these reduced-symmetry particles are similar to those of full nanoshells, however they may be more convenient to prepare.
\end{abstract}

Keywords: gold, optical properties, plasmon resonance

\section{Introduction}

The plasmon-induced optical absorption of nanoparticles of the coinage metals $(\mathrm{Cu}, \mathrm{Ag}$, and $\mathrm{Au})$ has attracted interest due to the potential application of this phenomenon in solar glazing [1,2], resonant fluorescence enhancement [3], medical treatment [4, 5], and optical sensing [6]. In general, these particles display one or more plasmon resonances with electromagnetic radiation of appropriate wavelengths. A variety of shapes such as nanoshells, nanorods, nano-caps, nano-cups, and triangles have been successfully synthesized, most commonly from gold or silver [7]. There has been considerable prior work 
Optical properties of suspensions of gold half-shells

in respect of the wavelength at which peak absorption occurs, and the manner in which this is controlled by shape. Absorption in the near-infrared is particularly attractive due to its possible technological applications. Half-shells, nano-caps and nano-cups are members of the topologically-related family of semi-shells [8], all of which exhibit red-shifted plasmon absorption relative to simple nanospheres. In prior work the optical properties of suspensions of discrete nano-caps and other semi-shells consisting of less than a hemisphere of metal[8, 9], and of contiguous coatings of half shells, nano-caps, and nano-cups on substrates have been reported[10-13], but there appears to have been no specific report yet of the optical properties of suspensions of discrete half-shells. This is the topic that we addressed here.

\section{Experimental procedure}

Densely packed templates of spherical particles have previously been successfully used for the fabrication of half-shells [12], nano-caps and nano-cups [8, 11], triangles [14, 15$]$, asymmetrical dimmers [16], and discs [17] by physical deposition of a metal. The problem with the fabrication of half-shells is the difficulty in subsequently separating the particles from one another, which normally causes inhomogeneity of shape and poor yield when attempts are made to prepare suspensions. In our work we solved this problem by spin-coating a glass substrate with a very dilute suspension of PSPs, so that they were not contiguously distributed on the substrate, as shown in Fig. 1. The density of the PSP layer can be controlled using the concentration of the particle suspension and the spinning speed used for spin-coating. Thermal metal deposition was then performed perpendicular to the sample substrate, followed by the removal of the polymer core with $\mathrm{CH}_{2} \mathrm{Cl}_{2}$ to obtain a suspension of hollow half-shells. This protocol has successfully avoided the problem of inter-connection between the half-shells that occurs with denselypacked templates of spheres (for example, as in the work of Love et al.[12]). Half-shells with nominal inner diameters of $50 \mathrm{~nm}, 100 \mathrm{~nm}, 150 \mathrm{~nm}, 200 \mathrm{~nm}$ and $400 \mathrm{~nm}$ were prepared in the present work. The thickness of the metal was fixed at $\sim 20 \mathrm{~nm}$ and was controlled using a quartz crystal microbalance. This provided samples with a range of aspect ratios, $R_{\mathrm{i}} / R_{\mathrm{o}}$, where $R$ is the inner or outer radius respectively. Micrographs of half- were taken in a Zeiss Supra 55VP scanning electron microscope using an in-lens 
Optical properties of suspensions of gold half-shells

detector. The optical extinction spectra of the half-shells suspended in $\mathrm{CH}_{2} \mathrm{Cl}_{2}$ were measured in a Cary 5E UV-visible-NIR spectrometer.

\section{Results}

A micrograph of half-shells taken before removal of the PSPs is shown in Fig. 2a, while examples of the particles produced after removal of the template particles are shown in Figs. 2b-d. Figs. $2 \mathrm{c}$ and $2 \mathrm{~d}$ show single shells with the convex and concave sides facing up respectively. It can be seen that the diameter of the half-shells is relatively smaller than that of the PSPs used for the template. This is due to the shrinkage caused by surface tension of the edge gold and the loss due to the breakage of the very thin gold coating layer along the equator of the PSPs. Gold coatings formed by thermal evaporation are generally not continuous if they are thinner than $20 \mathrm{~nm}$. This might explain why the edges of the halfshells are irregular. It can be seen from Fig. 2 that the surfaces of the half-shells are not smooth either, being characterized by numerous small gold grains. Surface roughness is expected to have a red-shifting influence of the plasmon resonance $[18,19]$. Some small nanometer-sized holes can also be observed around the surface of the shells despite the total thickness of deposited gold being $\sim 20 \mathrm{~nm}$.

The optical extinction spectra of the half-shells are shown in Fig. 3. In the case of half-shells made with $200 \mathrm{~nm}$ PSP spheres, a peak in extinction at $958 \mathrm{~nm}$ was observed, which can be contrasted to a peak obtained at $830 \mathrm{~nm}$ in previous work with asymmetric gold nano-caps [9]. This result is in agreement with the observation that the degree of symmetry of semi-shells also effects their plasmon resonances. Overall, it can be observed in Fig. 3 that the peak optical extinction red-shifts with the increasing half-shell diameter, that is, with an increasing ratio of inner to outer radius, and that it can be varied from about $530 \mathrm{~nm}$ to in excess of $2000 \mathrm{~nm}$ by this means. However, the bigger half-shells produce a broader extinction peak than the smaller ones, a phenomenon that has also been noted with true nanoshells [20] and which works mitigates somewhat against their use in spectrally selective applications [21].

The wavelength of the peak extinction of the half-shells is plotted in Fig. 4 against aspect ratio. Data calculated using the Bohren-Hoffman [22] algorithms for full gold nanoshells (not half-shells) in $\mathrm{CH}_{2} \mathrm{Cl}_{2}$, 
Optical properties of suspensions of gold half-shells

and of the same nominal inner and outer diameters, are superimposed. The surprising fact is not that the peak extinction of either shape depends so sensitively on aspect ratio (this is already well known for nanoshells and expected for half-shells) but rather that the half-shells follow a similar trend as the nanoshells. Of course the surface roughness present might also have contributed to the optical extinction spectra since this has a red-shifting effect $[18,23]$. Nevertheless, the dominant factors controlling the spectra in this case are evidently the diameter (scattering) and aspect ratio (absorption) of the particles.

\section{Discussion}

Although semi-shells may be prepared in a variety of elements by using template-assisted physical vapour deposition, it is those of gold and silver that have so far attracted the most attention. The reason is that these two elements are ideal when stable nanoparticles with a plasmon resonance in the visible or infrared wavelengths are sought. Nanoparticles of aluminium, the alkali metals, and copper also exhibit strong plasmon resonances [24] but are too susceptible to oxidation for general use. Most other elements have electron densities that are not appropriate for plasmon resonances in the vicinity of the visible wavelengths. The choice between gold or silver might come down to the position of the corresponding plasmon resonances. Nanoparticles of silver generally display optical extinction peaks in the blue to green half of the visible spectrum, whereas the gold nanoparticles have extinction maxima from green upwards into the near-infrared. It should be born in mind, however, that silver nanoparticles are not immune from oxidation under ambient conditions. Finally, there is a further attraction of gold: its surface chemistry which permits the facile attachment of organic molecules onto its surface. This is useful in instances where the nanoparticle must be coated with some functional chemical layer.

\section{Conclusions}

Hollow half-shells of controlled size have been successfully fabricated using a dispersed polystyrene particle template, followed by the thermal deposition of gold perpendicularly to the template. These particles could be readily separated and brought into suspension. The absorption peaks were found to red- 
Optical properties of suspensions of gold half-shells

shift with the increasing diameter and values of $R_{i} / R_{0}$. A dramatically large red-shift to $2080 \mathrm{~nm}$ has been observed for the half-shells which had a diameter of $400 \mathrm{~nm}$ and an aspect ratio of 0.91 .

\section{Acknowledgements}

The authors thank the Australian Research Council and the Industrial Physics Division of CSIRO, Australia for support, and acknowledge the useful advice of A/Prof. Mike Ford, University of Technology Sydney.

\section{References}


Optical properties of suspensions of gold half-shells

\section{Figure captions}

Fig. 1. (Color online). Schematic illustration showing how polystyrene particle (PSP) templates were used to produce discrete half-shells of gold.

Fig. 2. SEM micrographs of half-shells. a) Half-shells on $200 \mathrm{~nm}$ PSPs, attached to the glass substrate. b) Half-shells formed on $400 \mathrm{~nm}$ PSPs after separation. c) Single half-shell from (b) with convex side facing up. d) Single half-shell from (b) with concave side facing up.

Fig. 3. (Color online). The optical extinction spectra of suspensions of gold half-shells with nominal core diameters in nanometers as indicated, and a fixed shell thickness of $20 \mathrm{~nm}$.

Fig. 4. The wavelengths of maximum optical extinction of the gold half-shells plotted against their aspect ratios. The trend calculated for extinction due to the dipole plasmon resonance of hollow gold nanoshells in $\mathrm{CH}_{2} \mathrm{Cl}_{2}$ is superimposed. 
Optical properties of suspensions of gold half-shells

\section{FIGURES}

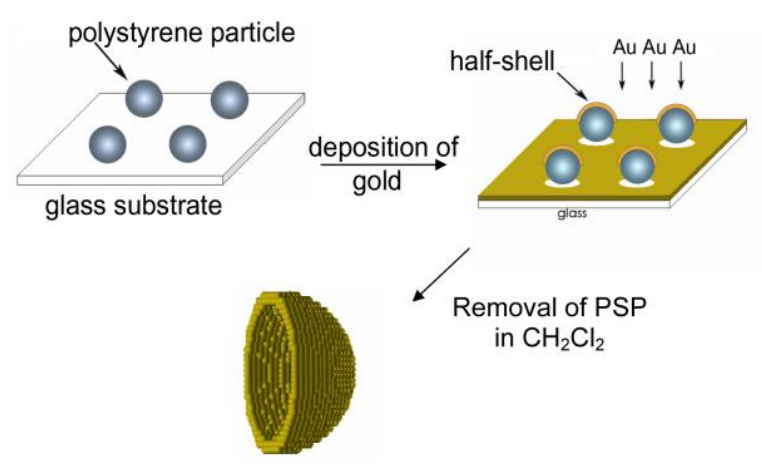

Fig. 1. (Color online). Schematic illustration showing how polystyrene particle (PSP) templates were used to produce discrete half-shells of gold.

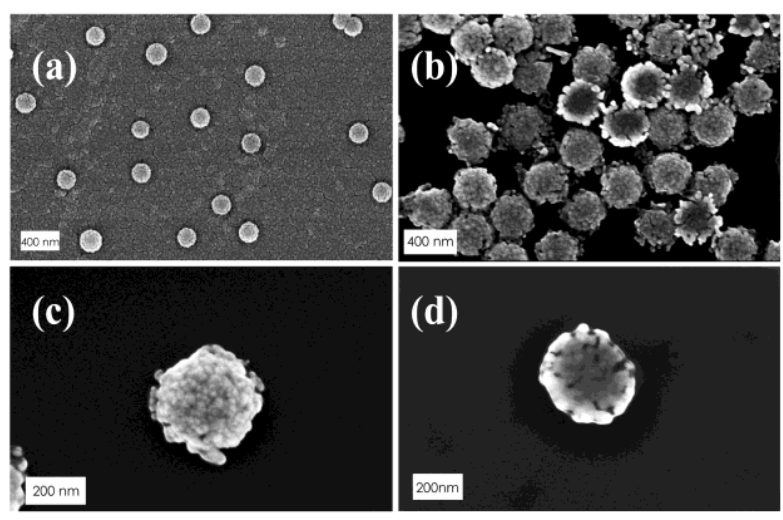

Fig. 2. SEM micrographs of half-shells. a) Half-shells on $200 \mathrm{~nm}$ PSPs, attached to the glass substrate. b) Half-shells formed on $400 \mathrm{~nm}$ PSPs after separation. c) Single half-shell from (b) with convex side facing up. d) Single half-shell from (b) with concave side facing up. 


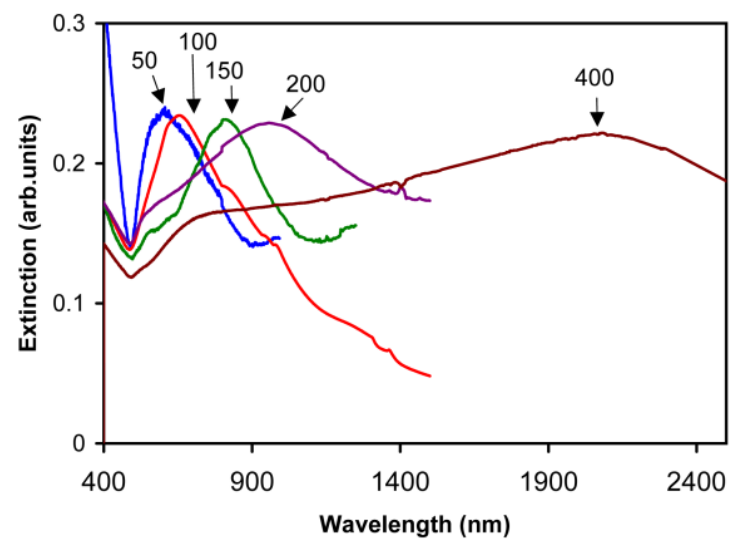

Fig. 3. (Color online). The optical extinction spectra of suspensions of gold half-shells with nominal core diameters in nanometers as indicated, and a fixed shell thickness of $20 \mathrm{~nm}$.

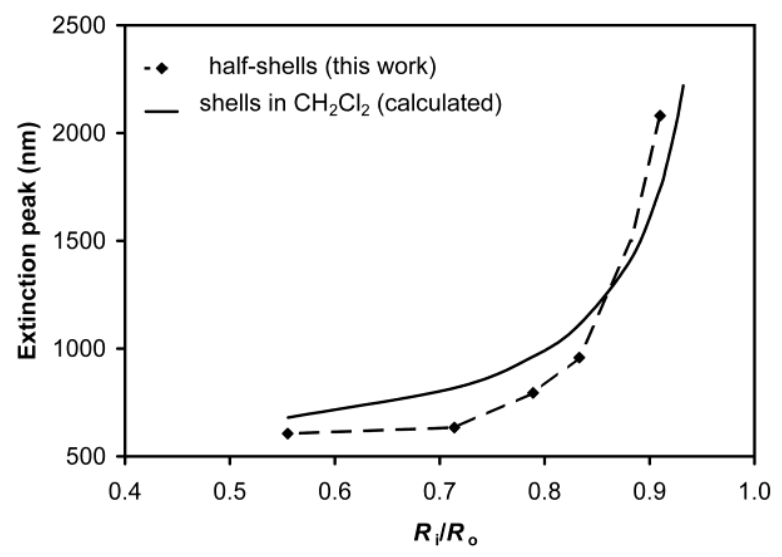

Fig. 4. The wavelengths of maximum optical extinction of the gold half-shells plotted against their aspect ratios. The trend calculated for extinction due to the dipole plasmon resonance of hollow gold nanoshells in $\mathrm{CH}_{2} \mathrm{Cl}_{2}$ is superimposed. 
Optical properties of suspensions of gold half-shells

$1 \quad$ S. Schelm and G.B. Smith, Appl. Phys. Lett., 82 (2003) 4346-4348.

2 X. Xu, M. Stevens and M.B. Cortie, Chem. Mater., 16 (2004) 2259-2266.

3 K. Aslan, Z. Leonenko, J.R. Lakowicz and C.D. Geddes, J. Phys. Chem. B, 109 (2005) 3157-3162.

4 C. Loo, A. Lin, L. Hirsch, M.H. Lee, J. Barton, N. Halas, J. West and R. Drezek, Technol. in Cancer Res. \& Treatment, 3 (2004) 33-40.

5 D.P. O'Neal, L.R. Hirsch, N.J. Halas, J.D. Payne and J.L. West, Cancer Lett, 209 (2004) 171-176.

6 C.J. Murphy, T.K. Sau, A.M. Gole, C.J. Orendorff, J. Gao, L. Gou, S.E. Hunyadi and T. Li, J. Phys. Chem. B, 109 (2005) 13857-13870.

7 K.L. Kelly, E. Coronado, L.L. Zhao and G.C. Schatz, J. Phys. Chem. B, 107 (2003) 668-677.

8 J. Liu, B. Cankurtaran, G. McCredie, M. Ford, L. Wieczorek and M. Cortie, Nanotechnology, 16 (2005) 3023-3028.

9 J. Liu, A.I. Maaroof, L. Wieczorek and M.B. Cortie, Adv. Mater., 17 (2005) 1276 - 1281.

10 H. Takei, M. Himmelhaus and T. Okamoto, Opt. Lett., 27 (2002) 342-344.

11 C. Charnay, A. Lee, S. Man, C.E. Moran, C. Radloff, R.K. Bradley and N.J. Halas, J. Phys. Chem. B, 107 (2003) 7327-7333.

12 J.C. Love, B.D. Gates, D.B. Wolfe, K.E. Paul and G.M. Whitesides, Nano Lett., 2 (2002) 891-894.

13 J. Liu, B. Cankurtaran, L. Wieczorek, M.J. Ford and M.B. Cortie, Adv. Func. Mater., 16 (2006) 1457-1461.

14 J.C. Hulteen and R.P. Vanduyne, J Vac Sci Technol A, 13 (1995) 1553-1558.

15 G. Zhang, D.Y. Wang and H. Mohwald, Nano Lett., 5 (2005) 143-146.

16 Y. Lu, H. Xiong, X.C. Jiang, Y.N. Xia, M. Prentiss and G.M. Whitesides, J. Am. Chem. Soc., 125 (2003) 12724-12725.

17 P. Hanarp, M. Kall and D.S. Sutherland, J. Phys. Chem. B, 107 (2003) 5768-5772.

18 H. Wang, G.P. Goodrich, F. Tam, C. Oubre, P. Nordlander and N.J. Halas, J. Phys. Chem. B, 109 (2005) 11083-11087.

19 A. Hoffmann, Z. Lenkefi and Z. Szentirmay, J Phys-Condens Mat, 10 (1998) 5503-5513.

20 R.D. Averitt, D. Sarkar and N.J. Halas, Phys. Rev. Lett, 78 (1997) 4217-4220.

21 S. Schelm and G.B. Smith, J. Opt. Soc. Am. A, 22 (2005) 1288-1292.

22 C.F. Bohren and D.R. Huffman, in Absorption and Scattering of Light by Small Particles, Wiley, 1998.

23 C.L. Nehl, N.K. Grady, G.P. Goodrich, F. Tam, N.J. Halas and J.H. Hafner, Nano Lett., 4 (2004) $2355-2359$.

24 M.G. Blaber, M.D. Arnold, N. Harris, M.J. Ford and M.B. Cortie, Physica B, (2007) in press. 
Optical properties of suspensions of gold half-shells 